TITRE: APPRENDRE À (RÉ)ÉCRIRE EN LISANT À L'ÉDUCATION DES ADULTES : UN DISPOSITIF EXPLOITANT LE CONTE MERVEILLEUX

Auteur(s): VAnessa Boily, M.A., Université du Québec À MontréAl, Conseillère pédagogique, PROJET CARREFOUR FGA

PUBLICATION: CRÉATION DE DISPOSITIFS DIDACTIQUES ET ENSEIGNEMENT-APPRENTISSAGE DIVERSIFIÉ EN LITTÉRATIE : VERS UNE VALORISATION DE LA RECHERCHE-DÉVELOPPEMENT ET DE LA RECHERCHEACTION EN ÉDUCATION

PAGES: $131-144$

Directeurs: Marie-Christine Beaudry, ISABElle CaRignan et FrançOIS LAROSE

ÉdiTEUR: LES ÉDITIONS DE L'UNIVERSITÉ DE SHERBROOKE, 2017

ISBN: 978-2-7622-0356-1

URI: HTTP://HDL.HANDLE.NET/11143/10129

DOI: HTTP://DX.DOI.ORG/10.17118/11143/10129 


\title{
APPRENDRE À (RÉ)ÉCRIRE EN LISANT À L'ÉDUCATION DES ADULTES : UN DISPOSITIF EXPLOITANT LE CONTE MERVEILLEUX
}

\author{
Vanessa Boily, M.A., Université du Québec à Montréal, \\ Conseillère pédagogique, Projet Carrefour FGA
}

Résumé : La recherche actuelle en didactique de l'écriture du français langue maternelle prône des méthodes plus diversifiées que l'enseignement canonique des genres (Lebrun, 2007). Dans le cadre de notre recherche exploratoire, nous avons expérimenté un dispositif didactique en réécriture, inspiré de celui de Sorin (2005). Nous avons modifié ce dernier afin de l'adapter à notre clientèle spécifique à l'éducation aux adultes. Notre dispositif prône un changement de postures estudiantine et enseignante (Sorin, 2005; Tauveron et Sève, 2005). L'analyse quantitative des résultats voulait vérifier l'efficacité de notre dispositif (objectif 1 ) et l'analyse qualitative des rédactions des élèves et des entrevues semi-dirigées s'est concentrée sur le potentiel de notre dispositifà permettre aux élèves de mettre en oeuvre un projet d'auteur (objectif 2).

Mots-clés : réécriture, posture d'auteur, dispositif didactique, raccrocheurs, éducation aux adultes, conte. 


\section{Introduction}

Pour développer la compétence à écrire des élèves, de récentes recherches en didactique de l'écriture du français langue maternelle prônent des méthodes plus diversifiées que l'enseignement canonique des genres, comme la réécriture (Baptiste, 2005; Lebrun, 2007; Sorin, 2005). Pour répondre à ce besoin didactique, nous avons créé un dispositif axé sur la réécriture inspiré de celui de Sorin (2005).

L'intérêt de notre étude se situe aussi dans la clientèle que nous avons étudiée (Boily, 2014). Nous avons expérimenté notre dispositif auprès de raccrocheurs, plus précisément des élèves inscrits dans un cours de français de la quatrième et de la cinquième secondaire dans un centre d'éducation des adultes dans la région de Montréal. L'expérimentation s'est tenue lors d'un atelier sur le conte destiné à ces élèves raccrocheurs. Peu d'outils didactiques sont destinés spécifiquement aux raccrocheurs alors que tout doit être mis en œuvre pour les aider et pour réduire leur vulnérabilité sur les plans social et économique (Lepitre, 2007; MEQ, 2004). Nous proposons donc un dispositif didactique en écriture littéraire adapté à cette clientèle particulière.

Pour favoriser la réussite et la persévérance scolaires, il faut, entre autres, développer davantage la compétence à écrire (Chartrand, 2009). Pour ce faire, le ministère de l'Éducation, du Loisir et du Sport (MÉLS, 2008) reconnait que les enseignants ont besoin de plus de formations et d'outils en ce sens. La présente recherche s'inscrit dans le courant des études visant à mettre de l'avant l'écriture littéraire en classe, plus précisément la réécriture, pour développer les compétences scripturales des élèves (Reuter, 2009; Sorin, 2005; Tauveron et Sève, 2005), ici des raccrocheurs.

Nous voulions répondre aux questions de recherche suivantes: 1) Quelle est l'efficacité d'un dispositif didactique alliant lecture littéraire et réécriture sur les productions écrites des élèves raccrocheurs? et 2) Dans quelle mesure le dispositif testé permet-il aux élèves d'adopter une posture d'auteur?

En comparant les résultats de notre prétest et de notre postest, nous ne pouvons pas affirmer l'efficacité de notre dispositif, de nombreux biais étant reconnus. Néanmoins, l'analyse des entrevues réalisées à la suite du postest avec les participants révèle que tous présentaient un projet d'auteur, mais que l'élaboration de ce dernier variait grandement d'un élève à l'autre. Cette analyse fine des profils de scripteurs à la suite de notre dispositif est très encourageante pour la recherche en écriture littéraire, bien qu'une étude empirique soit toujours nécessaire pour affirmer ses effets hors de tout doute. 


\section{Problématique}

Notre problématique est double : elle agit sur un axe social, le décrochage scolaire (Larose et Boulanger, 2013), et sur un axe didactique (Sorin, 2005).

\section{Un phénomène social préoccupant : le décrochage scolaire}

Au regard des statistiques, le décrochage scolaire est un phénomène social de poids : en 20092010, 8,5\% des jeunes âgés de 20 à 24 ans étaient considérés comme des décrocheurs (Gilmore, 2010). Cela signifie que leur diplôme d'études secondaires n'était pas terminé et qu'ils n'étaient pas inscrits dans un établissement scolaire. En effet, de nombreux jeunes profitent de la formation générale des adultes pour terminer leurs études secondaires. Environ $40 \%$ des jeunes femmes et de $20 \%$ à $30 \%$ des jeunes hommes décrocheurs retournent à l'école avant l'âge de 25 ans (Raymond, 2008). Mais que pouvons-nous faire pour stimuler cette reprise des études et pour mener les élèves vers la réussite scolaire?

Peu d'études étudient les jeunes raccrocheurs, et encore moins sont axées sur les mesures didactiques possibles en classe de français à la formation générale des adultes (FGA), la plupart abordant les causes et les conséquences du décrochage (Raymond, 2008). PARcours (Pratiques d'accompagnement du raccrochage scolaire des 16-20 ans) est un organisme qui a été créé spécifiquement pour soutenir les acteurs du milieu qui travaillent auprès des jeunes raccrocheurs, une population étudiante en croissance. Dans un récent colloque mené par cet organisme en 2012, l'ensemble des acteurs impliqués a soulevé l'importance de différencier les pratiques pour les raccrocheurs. Les programmes alternatifs fonctionnent bien en offrant variété, soutien et flexibilité. L'originalité des projets touche aussi les jeunes raccrocheurs. Le MÉQ (2004) a déjà fait ce constat, puisque les jeunes raccrocheurs ont un profil bien à eux (Janosz, 2002), ce qui mènerait à une différenciation des approches promues par les centres de formation (Lepitre, 2007).

\section{Étudier plus avant la réécriture au secondaire}

Pour accroitre la réussite scolaire, la compétence à écrire est un facteur déterminant (Chartrand, 2009). De nouvelles pratiques axées sur la créativité sont prônées par la recherche en didactique de l'écriture littéraire, dont la réécriture est une forme (Sorin, 2005; Tauveron et Sève, 2005). Des études en salle de classe semblent montrer que la réécriture est bénéfique pour le développement de la compétence à écrire des élèves (Reuter, 2009). En Europe, une étude de Bessonnat (2000) auprès d'élèves français de troisième (équivalent de la troisième secondaire au Québec) a révélé que les activités de réécriture répétées ont permis de modifier la représentation qu'avaient les élèves du processus de création, vers une représentation plus ouverte et moins figée. À l'université, Houdart-Mérot (2008) a pour sa part noté une plus forte compréhension de l'intertextualité et de sa place dans la 
création et dans l'interprétation d'une œuvre chez ses étudiants. Au primaire, Chénard-Guay (2010) a relevé que les élèves avaient une meilleure analyse des textes lus à la suite des activités de réécriture. Cependant, d'autres études sont nécessaires pour dégager tous les effets de cette pratique (Bessonnat, 2000; Houdart-Mérot, 2008; Tauveron, 2005). Seules Beaudry et Huneault (2011) auraient expérimenté l'écriture d'invention au Québec, et ce, au niveau secondaire, mais leur objet d'étude était la lecture. La posture de scripteur lors de la réécriture, telle que nous voulons l'étudier ici, ne constituait pas l'objet examiné.

Ces constats nous ont amenés à établir deux objectifs pour notre recherche:

1. Vérifier l'efficacité d'un dispositif didactique portant sur la réécriture au secondaire, et ce, avec une clientèle peu étudiée, les jeunes raccrocheurs.

2. Vérifier si notre dispositif didactique permet à nos participants d'adopter une posture d'auteur, et ce, à l'aide de deux indicateurs (Tauveron et Sève, 2005).

\section{Cadre conceptuel}

Parmi les concepts autour desquels gravite notre recherche, deux sont primordiaux : la réécriture elle-même et la posture estudiantine lui étant associée. Le conte merveilleux se doit aussi d'être défini comme notre dispositif repose entièrement sur ce genre littéraire.

\section{La réécriture : une pratique didactique intertextuelle}

La réécriture est une forme d'écriture littéraire (Petitjean, 2003). Cette forme d'écriture entretient un lien fort avec la lecture littéraire (Sorin, 2005; Tauveron et Sève, 2005), puisqu'elle imite ou transforme un texte lu au préalable (Petitjean, 2003). Imiter, c'est reprendre considérablement les éléments du texte source lu (hypotexte); ce qui est l'équivalent de la réécriture actualisée (Gervais et Hinz, 2007). De son côté, transformer, c'est modifier davantage l'hypotexte; ce qui est l'équivalent de l'adaptation (Gervais et Hinz, 2007). L'intertextualité représente les liens existants entre divers textes : l'auteur part donc d'un hypotexte pour créer un nouveau texte (hypertexte) en conservant certains éléments du premier texte (liens communs ou intertextuels) (Genette, 1982). La place de l'intertextualité est donc centrale dans la mise en place de la pratique didactique qu'est la réécriture (voir figure 3.1). 


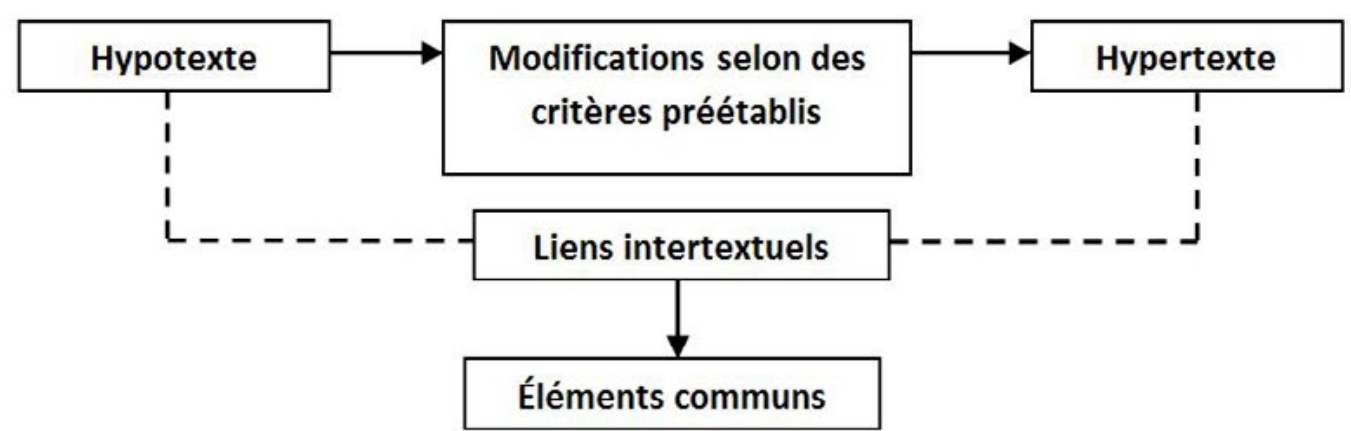

Figure 1. Schéma inspiré de Genette (1982) représentant les concepts en jeu lors de la réécriture

D’ailleurs, pour Houdart-Mérot, la lecture littéraire mise à profit lors d'une réécriture implique que « l'interprétation d'un texte littéraire s'appuie presque toujours sur une lecture intertextuelle » (2008, p. 129).

\section{Une nouvelle posture}

La réécriture nécessite un changement de posture, c'est-à-dire qu'elle exige de l'élève qu'il se positionne en tant qu'auteur, ayant un projet d'auteur, et non plus comme un simple scripteur écrivant pour son enseignant (Lebrun, 2007). Tauveron et Sève expliquent ainsi la posture auctoriale en contexte scolaire: "Dans les classes où nous avons travaillé, le contrat stipule que les élèves, après avoir exploré les règles de jeu construites par les auteurs légitimes pour des lecteurs coopératifs, échangent les rôles dans la communication littéraire et construisent à leur tour des aires de jeu pour des lecteurs aussi « intelligents », « actifs », " sensibles » et « cultivés » qu'ils furent eux-mêmes dans la partie et position précédente $(2005$, p. 22).

Pour ce faire, il faut que chaque élève ait à sa disposition un répertoire intériorisé de procédés stylistiques et littéraires et qu'il utilise ce répertoire dans son texte en faisant des choix d'auteur (premier indicateur d'un projet d'auteur). Il faut aussi qu'il ait conscience de l'effet désiré chez son lecteur modèle (Eco, 1989) - après l'avoir identifié - (deuxième indicateur d'un projet d'auteur). La présence de ces deux indicateurs révèlerait un projet d'auteur, une posture d'auteur chez l'élève selon Tauveron et Sève (2005).

\section{Le conte merveilleux}

Dans le cadre de notre recherche, le genre littéraire abordé est le conte merveilleux. "Les critères de définition des genres sont hétérogènes : critères formels parfois (présence d'une superstructure 
textuelle particulière, présence de certaines formules, comme dans le conte merveilleux), critères thématiques souvent [...], cadre pragmatique [...]»(Crinon, 2006, p. 17). En ce sens, le conte merveilleux est un texte de type narratif, c'est-à-dire que son but premier est de raconter une histoire, et celle-ci est fictive. Propp (1970), en présentant la morphologie du conte, insiste sur ses invariants, qui se succèdent toujours dans le même ordre, même s'ils ne sont pas tous présents d'une histoire à l'autre. Par exemple, la présence d'un manque ou d'un méfait qui mène à un départ et qui se termine par un mariage entre les personnages constitue une suite d'invariants du conte merveilleux (Propp, 1970).

Une étude ayant souligné l'échec de l'enseignement traditionnel du conte (Crinon, 2006), nous proposons une façon novatrice de l'enseigner aux élèves. De plus, le conte étant un genre de texte souvent lu dès la petite enfance (Rufiange, 2008), cela pourrait contribuer à augmenter les connaissances préalables des élèves à son sujet, surtout intertextuelles, et faciliter la mise en place de notre dispositif (Sorin, 2005; Tauveron et Sève, 2005).

\section{Méthodologie}

\section{Les sujets}

Notre échantillon est considérablement réduit, ce qui est un biais avoué de notre recherche : sept participants sur douze ont complété l'ensemble des activités. La clientèle plus volatile avec laquelle nous travaillons peut expliquer ce biais (MÉQ, 2004). Nos participants sont principalement des raccrocheurs inscrits à une école de FGA de Montréal en fréquentation de jour, et ce, en quatrième ou cinquième secondaire en français. Ils se sont portés volontaires pour participer à un atelier siglé sur le conte merveilleux (notre dispositif didactique), à raison de deux heures deux fois par semaine pour sept semaines (pour un total de 27 heures). Les ateliers ont été animés par l'enseignante chercheure, qui a quatre années d'expérience en enseignement à la FGA et qui a aussi enseigné au secteur jeune auparavant.

\section{Le dispositif didactique}

Adapté d'un dispositif de réécriture créé par Sorin (2005) et destiné aux élèves du primaire, notre dispositif se déploie en six étapes, dont trois (étapes 2, 3 et 4) pouvant être répétées un nombre X de fois en fonction des besoins des apprenants :

1. L'écriture d'un premier conte selon une consigne donnée par l'enseignant;

2. Lire une œuvre riche en intertextualité;

3. Analyser l'œuvre avec les élèves afin de mettre en évidence les traces d'intertextualité; 
4. Réécriture d’un conte connu (en partie ou en totalité);

5. L'écriture d'un dernier conte selon la même consigne qu'à la première étape;

6. Retour sur la tâche d'écriture par une discussion élève-enseignant (entrevues).

Les textes choisis devaient recéler de traces intertextuelles décelables pour les élèves : les contes classiques des frères Grimm et les versions de Perrault et d'Andersen, mais aussi des versions internationales de ces classiques (Morel, 2008) et des contes mythologiques les ayant inspirés. Les élèves étaient amenés à discuter des œuvres lues et à énoncer diverses interprétations; le but étant toujours de développer un répertoire de procédés stylistiques et littéraires chez les apprenants pour qu'ils deviennent des auteurs avec un projet d'écriture à effet esthétique conscient pour un lecteur modèle (ECo, 1989).

\section{Les instruments de collecte de données et l'analyse des données}

Afin de répondre à nos deux objectifs de recherche, deux instruments de collecte de données ont été employés : un prétest et un postest (premier instrument) et une entrevue semi-dirigée (deuxième instrument).

Le prétest et le postest ont été administrés lors des étapes 1 et 5 avec la même consigne:

Vous devez écrire un conte merveilleux d'au moins 350 mots en vous basant sur ce que vous savez déjà de ce genre de texte. Vous avez trois heures pour le faire. Bonne rédaction! Cet instrument vise à vérifier l'efficacité du dispositif ( $1^{\text {er }}$ objectif). Toutefois, l'absence de groupe de comparaison et la taille réduite de notre échantillon limitent grandement la portée de nos résultats quantitatifs. Pour analyser les résultats obtenus au prétest et au postest, nous avons employé une grille de codification du conte critériée (A, B, C, D, E) - que nous avons préalablement pilotée - et pondérée (20 points par critère). Les cinq critères évalués étaient les éléments intertextuels, l'originalité, les éléments merveilleux, l’organisation stratégique ainsi que la continuité et la progression.

Le second instrument est une entrevue réalisée lors de l'étape 6 du dispositif. Nous avons posé sept questions aux élèves afin de déterminer si une posture d'auteur était observée à la suite de l'implantation du dispositif (2e objectif). Voici le classement initial des projets d'auteur que nous avons établi, en fonction des deux indicateurs de Tauveron et Sève (2005) présentés dans le cadre conceptuel :

1. Présence : conscience des choix opérés (CCO, premier indicateur) et lecteur modèle anticipé (LMA, deuxième indicateur)

2. Balbutiement: un des deux indicateurs seulement

3. Absence: Aucun indicateur 
Une étude de contenu a été réalisée sur les verbatims des entrevues pour déceler les échanges avec les élèves qui révèlent un projet d'auteur conscient. Notre codification s'est effectuée dans un premier temps en fonction des indicateurs de Tauveron et Sève (2005). Dans un deuxième temps, nous avons croisé ces résultats avec l'analyse des productions écrites.

\section{Résultats}

Bien qu'ils doivent être relativisés, nos résultats décèlent un certain impact de notre dispositif sur nos élèves ainsi que la présence d'un projet d'auteur chez ces derniers lors du postest.

\section{Impact du dispositif axé sur la réécriture}

En comparant les résultats obtenus au prétest et au postest, une nette amélioration est notable pour plusieurs critères, principalement pour les éléments intertextuels et merveilleux ainsi que pour la continuité et la progression (voir le tableau 5.1). Cependant, l'échantillon restreint de sept participants limite grandement la portée de ces résultats; parfois, il ne s'agit que d'un participant qui s'est amélioré. Par exemple, l'élève 1 a vu son résultat passer de 4 à 20 sur 20 et l'élève 7, de 8 à 20 sur 20 au postest pour le critère portant sur l'intertextualité.

Tableau 5.1 Comparaison des moyennes obtenues pour chaque critère au prétest et au postest

\begin{tabular}{|l|l|l|}
\hline Critères & Moyennes au prétest & Moyennes au postest \\
\hline Éléments intertextuels & 14.86 & 19.43 \\
\hline Originalité & 18.86 & 20 \\
\hline Éléments merveilleux & 17.14 & 19.43 \\
\hline Organisation stratégique & 16 & 17.71 \\
\hline Continuité et progression & 14.86 & 17.14 \\
\hline
\end{tabular}

Il est toutefois intéressant de noter que, sans que la consigne ait changé, les contes du postest présentent davantage de traces intertextuelles riches et habiles que ceux du prétest. En voici un exemple qui provient du texte de l'élève 1 qui a fait une réécriture du conte Le Prince Grenouille : 


\section{La dame chenille}

Dans un royaume sans roi vivait une dame affreuse ayant l'apparence d'une vieille chenille. Cette dame passait ses journées à marcher à travers le royaume en direction de sa maison, mais le chemin était long et elle était faible et fatiguée; la vieille dame n’y était jamais parvenue.

$[\ldots]$

Et alors, sans attendre l'approbation de l'homme, elle l'embrassa et puis, soudain, dans un éclat de lumière, la dame chenille se métamorphosa en jolie princesse.

Un élément intéressant dans ce texte est l'inversion (Tauveron et Sève, 2005, p. 111) : l'élève 1 a inversé les rôles de la princesse et du prince. Ici, le prince n'est pas grenouille, mais un pauvre généreux qui aidera la vieille dame chenille (qui se métamorphosera ensuite en belle princesse) alors que, dans le conte initial, le prince grenouille était celui qui changeait. Autre élément inusité, le pauvre homme ne change pas vraiment physiquement, malgré le fait que la dame chenille le trouvait repoussant au départ; ses bonnes valeurs reconnues en font un être nouveau aux yeux de la princesse, digne de diriger son royaume. L'élève aurait pu se limiter à changer le Prince Grenouille en Prince Chenille et reprendre la même trame narrative, mais il l'a modifiée plus avant en interchangeant les rôles des héros et en intégrant une nouvelle morale. Les traces intertextuelles présentées sont riches; nos résultats prennent donc leur sens dans une analyse plus qualitative que quantitative.

\section{Diverses postures d'auteur}

Pour l'ensemble de nos sept participants, un projet d'auteur a été relevé durant l'entrevue. C'est l'élaboration de ce dernier qui varie. Le tableau 5.2 relève le nombre de fois qu'un choix (CCO, premier indicateur) ou qu'un lecteur modèle (LMA, deuxième indicateur) a été mentionné par l'auteur du conte lors de l'entrevue, en plus de donner un exemple de réponse d'élève pour chaque code. En traitant uniquement les réponses à l'entrevue, deux élèves présentent un seul indicateur (Tauveron et Sève, 2005), le CCO, et donc se classent comme ayant un balbutiement de projet d'auteur. Pour l'élève 4, un possible manque de métalangage peut avoir biaisé notre classement (Beaudry et Huneault, 2011), car son texte témoigne d'une prise en compte exceptionnelle du destinataire. Nous le classerons donc comme présentant un projet d'auteur lors de notre deuxième temps d'analyse. Les cinq autres élèves avaient un projet d'auteur complet, c'est-à-dire que les codes CCO et LMA étaient présents dans leur entrevue. 
Tableau 5.2 Deux indicateurs du projet d'auteur

\begin{tabular}{|c|c|c|c|c|c|c|c|c|}
\hline Élève & (Exemple de réponse) & 1 & 2 & 3 & 4 & 5 & 6 & 7 \\
\hline cco & $\begin{array}{l}\text { Puis je voulais qu'elle puisse lui rendre service en mon- } \\
\text { trant aux autres que, dans le fond, ce n'était pas une } \\
\text { sorcière. [...] Bien une morale? C'est plutôt que ... c'est } \\
\text { un peu l'histoire, tu sais, de laide et belle (Gratelle au } \\
\text { bois mordant). Il n'y a personne qui est laid et la beauté } \\
\text { est juste un prototype et c'est la même chose vraiment } \\
\text { (Élève 2, question 2). }\end{array}$ & 5 & 5 & 12 & 2 & 4 & 2 & 3 \\
\hline LMA & $\begin{array}{l}\text { Au milieu, je n'avais pas d'idée parce que je voulais } \\
\text { rendre l'idée... Mon idée de base, c'était vraiment que } \\
\text { la personne qui lit sente que la valeur c'est vraiment } \\
\text { qu'on accepte d'aider gratuitement. Je ne sais pas. } \\
\text { Généreusement (Élève 1, question 2). }\end{array}$ & 2 & 2 & 5 & 0 & 5 & 0 & 2 \\
\hline
\end{tabular}

Étant donné qu'il n'y a pas eu d'entrevue réalisée au départ, il est impossible de statuer sur un changement de posture, de scripteur à auteur; il s'agit d'une faille méthodologique reconnue de notre projet. Néanmoins, il est tout de même possible de statuer que notre dispositif a permis aux élèves de vivre leur projet d'auteur.

Par ailleurs, un classement plus fin que celui de présence ou d'absence de projet est possible. Parmi les six élèves présentant les deux indicateurs du projet d'auteur, trois faisaient montre d'une réflexion plus approfondie sur leur processus d'écriture (élèves 3,5 et 7). Cette réflexion s'accompagnait notamment de l'explication de leurs choix (Tauveron et Sève, 2005) et de leur style d'écriture ou de leurs habitudes scripturales (Barthes, 1953 et 1972). Il s'agit donc d'une quatrième catégorisation possible du projet d'auteur, une classe à ajouter au-dessus de celles présentées précédemment (voir 4.3).

D’autres éléments sont ressortis des entrevues. Entre autres, l'expérience de lecture réalisée avant la réécriture est importante puisque les élèves doivent lire des œuvres marquantes (Tauveron et Sève, 2005) pour s'impliquer davantage en réécriture. L'entrevue nous donne un accès privilégié à cette bibliothèque intérieure (Louichon et Rouxel, 2010) en plus de nous révéler la présence ou l'absence du projet d'auteur. Par ailleurs, deux œuvres fortement intertextuelles lues en classe ont été souvent mentionnées en entrevue : Le délire de Somerset et Gratelle au bois mordant. Elles ont été marquantes pour nos participants (Tauveron et Sève, 2005).

Autre fait à noter, l'intertextualité a été source de stratégies pour plusieurs élèves. Conséquemment, l'enseignant doit amener les élèves «à identifier les moyens convoqués pour produire les effets perçus et à rapporter ces moyens à une intention d'auteur inscrite dans le texte » (Tauveron et Sève, 2005, p. 21) afin que les élèves puissent s'en servir à leur tour dans leurs textes. 


\section{Conclusion}

Aux deux questions que nous nous étions posées, nous ne pouvons répondre entièrement. Quelle est l'efficacité d'un dispositif didactique alliant lecture littéraire et réécriture sur les productions écrites des élèves raccrocheurs? La désaffection de participants et l'absence hors de notre contrôle de groupe de comparaison limite la portée de nos résultats bien que les sept participants aient obtenu une note supérieure au postest. Dans quelle mesure le dispositif testé permet-il aux élèves d'adopter une posture d'auteur? Les sept participants ont présenté un projet d'auteur; seulement sa complexité ou son élaboration variait. Nous pouvons maintenant présenter un classement plus précis des projets d'auteur des élèves que celui de Tauveron et Sève (2005).

D’autres études avec une méthodologie différente et des prises de données plus régulières sont nécessaires pour vérifier l'efficacité de la réécriture en classe, même avec notre clientèle cible que sont les raccrocheurs. Toutefois, nos résultats sont encourageants et s'inscrivent dans le courant visant la persévérance scolaire à l'aide d'initiatives locales innovantes et variées visant à briser par moments le modèle très traditionnel de l'éducation des adultes (MÉLS, 2004; PARcours, 2012). 


\section{Références}

Baptiste, L. (2005). Apprendre à écrire dans les programmes 2002 de l'école élémentaire : le recours à la littérature. Les sciences de l'éducation - Pour l'Ére nouvelle, 38(4), 133-150.

Barthes, R. (1953 et 1972). Le degré zéro de l'écriture. France : Éditions du Seuil.

Beaudry, M.-C. et Huneault, M. (2011). Étude de l'écriture d'invention pour développer la posture de lecteur littéraire chez des élèves de la cinquième secondaire. Revue pour la recherche en éducation, Acfas, Actes du colloque, 10 au 14 mai 2010, Université de Montréal.

Bessonnat, D. (2000). Une année de réécriture en troisième. Pratiques, (105/106), 83-110.

Boily, V. (2014). Apprendre à (ré)écrire aux raccrocheurs grâce à la lecture (Mémoire de maitrise). Université du Québec à Montréal.

Chartrand, S. G. (2009). Améliorer la maitrise de la langue écrite : la responsabilité de tous les enseignants. [Document PDF] Repéré à http://www1.mels.gouv.qc.ca/sections/prprs/pdf/prprsFiche23.pdf

Chénard-Guay, C. (2010). Écrire à la manière d'un auteur au premier cycle du primaire : pratiques et impacts (Mémoire de maitrise). Université de Montréal.

Crinon, J. (2006). L'écriture littéraire et les genres. Le français aujourd'hui, 2(153), 17-24.

Eco, U. (1989). Lector in Fabula : le rôle du lecteur. Paris, France : Éditions Grasset et Fasquelle.

Genette, G. (1982). Palimpsestes : la littérature au second degré. Paris, France : Seuil.

Gervais, F. et Hinz, C. (2007). Réécriture et musique dans les Contes de Grimm et Les Mille et une Nuits ». Dans F. Gervais et M. Noël-Gaudreault (dir), Littérature de jeunesse et espaces identitaires (p.75-81). Allemagne : Electronic Publishing Osnabrück.

Gilmore. J. (2010). Tendance du taux de décrochage et des résultats sur le marché du travail des jeunes décrocheurs. Canada : Statistique Canada. Repéré à http://www.statcan.gc.ca/pub/81-004-x/2010004/article/11339-fra.htm

Houdart-Mérot, V. (2008). Atelier de réécriture et critique littéraire en acte à l'université. Recherches et Travaux, (73), 125-137.

Janosz, M. (2002). Le profil des jeunes décrocheurs. Forum régional de l'lle de Montréal sur la persévérance, la réussite scolaire et le soutien aux raccrocheurs. Actes du forum du 23 mai 2002, Montréal. Québec : Les publications du Québec.

Larose, F. et Boulanger, D. (2013). Du communautaire au socioéducatif. Rapport de la recherche évaluative du programme «L'École des parents » déployé par le Regroupement économique et social du sud-ouest (RESO). Synthèse, faits saillants et recommandations. Sherbrooke/Québec: Université de Sherbrooke, Centre de recherche sur l'intervention éducative et socioéducative / Centre de transfert pour la réussite éducative du Québec. 
Lebrun, M. (2007). Rapport à l'écriture, posture auctoriale et ouverture culturelle. Revue des sciences de l'éducation, 33(2), 383-399.

Lepitre, J. (2007). Étude à posteriori du projet de raccrochage scolaire : Alternative scolaire à travers le modèle de motivation en contexte scolaire de Rolland Viau (Mémoire de maitrise). Université du Québec à Montréal.

Louichon, B. et Rouxel, A. (2010). Du corpus scolaire à la bibliothèque intérieure. Rennes : Presses universitaires de Rennes.

Ministère de l'Éducation, du Loisir et du Sport (MELS). (2008). La formation à l'enseignement: les orientations relatives à la formation en milieu de pratique. [Document PDF]. Québec : Gouvernement du Québec. Repéré à http://www.mels.gouv.qc.ca/fileadmin/site_web/documents/ reseau/formation_titularisation/FormationEnsFormMilieuPratique_f.pdf

Ministère de l'Éducation (MEQ). (2004). Le plaisir d'apprendre: J'embarque quand ça me ressemble. Québec: Ministère de l'Éducation.

Morel, F. (2008). Les histoires du Petit Chaperon rouge racontées dans le monde. Paris : Syros.

Petitjean, A. (2003). Écriture d'invention et nouveaux programmes des lycées. Écriture et invention. Actes du colloque d'Arras, IUFM de Nord-Pas-de-Calais.

PARcours. (2012). La grande rencontre 2012. $1^{\text {er }}$ et 2 novembre 2012, Université du Québec à Montréal. Repéré à http://parcours.uqam.ca/quoi-de-neuf/1-quoi-de-neuf/140-la-grande-rencontre-2012-sur-video.html

Propp, V. (1970). Morphologie du conte. Paris : Seuil.

Raymond, M. (2008). Décrocheurs du secondaire retournant à l'école. Culture, tourisme et Centre de la statistique de l'éducation : documents de recherche, (055), produit nº 81-595-M au catalogue de Statistique Canada.

Reuter, Y. (2009). Les récits de fiction en dernière année du primaire. Éléments d’analyse et de comparaison selon les pédagogies. Dans J.-L. Dufays et S. Plane (dir.), L'écriture de fiction en classe de français (p. 69-85). Namur: Presses universitaires de Namur.

Rufiange, C. (2008). Les modèles novateurs à l'école. Lurelu, 31(l), 99-100.

Sorin, N. (2005). Vers une didactique de l'écriture littéraire du récit de fiction au primaire. Nouveaux cahiers de la recherche en éducation, 8(1), 65-78.

Tauveron, A.-M. (2005). Accompagner l'écriture d'invention en classe. Pratiques, (127-128), 230-248.

Tauveron, C. et Sève, P. (2005). Vers une écriture littéraire ou comment construire une posture d'auteur à l'école de la GS au CM. Paris, France: Hatier. 